\title{
Chiral Particle Decay of Heavy-Light Mesons in a Relativistic Potential Model
}

\section{Takayuki Matsuki*}

Tokyo Kasei University, 1-18-1 Kaga, Itabashi, Tokyo 173-8602, JAPAN

E-mail: matsukietokyo-kasei.ac.jp

\section{Koichi Seo}

Gifu City Women's College, 7-1 Hitoichiba-Kitamachi, Gifu 501-0192, JAPAN

E-mail: seolgifu-cwC.ac.jp

Partial decay widths of the heavy-light mesons, $D, D_{s}, B$, and $B_{s}$, emitting one chiral particle ( $\pi$ or $K$ ) are evaluated in the framework of a relativistic potential model. Decay amplitudes are calculated by keeping the Lorentz invariance as far as possible and use has been made of the Lorentz-boosted relativistic wave functions of the heavy-light mesons. New formulation for the decay amplitude is given.

One of predictions of our calculation is very narrow widths of a few $\mathrm{keV}$ for yet undsicovered $B_{s}\left(0^{+}, 1^{+}\right)$mesons corresponding to ${ }^{2 S+1} L_{J}={ }^{3} P_{0}$ and ${ }^{33} P_{1}$ " assuming their masses to be 5617 and $5682 \mathrm{MeV}$, respectively, as calculated in our former paper. Here double quotation marks denote that they are mixed states between ${ }^{3} P_{1}$ and ${ }^{1} P_{1}$ and the dominant states are expressed by those without quotations.

In the course of our calculation, new sum rules are discovered on the decay widths in the limit of $m_{Q} \rightarrow \infty$. Among these rules, $\Gamma\left(D_{s 0}^{*}(2317) \rightarrow D_{s}+\pi\right)=\Gamma\left(D_{s 1}(2460) \rightarrow D_{s}^{*}+\pi\right)$ and $\Gamma\left(B_{s 0}^{*}(5615) \rightarrow B_{s}+\pi\right)=\Gamma\left(B_{s 1}(5679) \rightarrow B_{s}^{*}+\pi\right)$ are predicted to hold with a good accuracy.

The axial-vector coupling constant $\hat{g}=0.577$ used in our paper, which is derived by fitting our decay widths with experiments, is in a good agreement with other calculations, e.g., those derived by the leptonic decay width using the Schwinger-Dyson equation and the lattice QCD. This coupling may be used for the study of dissociation processes $\pi+J / \psi \rightarrow D+\bar{D}$ or $\pi+\Upsilon \rightarrow B+\bar{B}$ by exchanging $D^{*}$ or $B^{*}$.

Sixth International Conference on Quarks and Nuclear Physics,

April 16-20, 2012

Ecole Polytechnique, Palaiseau, Paris

\footnotetext{
*Speaker.
} 


\section{Formulation of Lorentz Invariant Decay Amplitude}

The decay processes of the heavy-light systems have been extensively studied by many people.[1] Most of them semi-relativistically treat hadrons and the overlap of heavy mesons is estimated using their meson wave functions in their own models. Assuming the emitted chiral meson/photon is expressed by the plain wave, $e^{-i k x}$, people normally adopts the following expression as the decay amplitude,

$$
\int d^{3} x \operatorname{Tr}\left(\psi_{X^{\prime} f}^{\dagger} O e^{i k x} \psi_{X i}\right)
$$

without any phases in the initial and final states, $\psi_{X i}$ and $\psi_{X^{\prime} f}$ of the heavy-light mesons $X^{(\prime)}$.

However, we have a phase factor in our relativistic wave function which is defined as,

$$
<0\left|q_{i}^{c}(\vec{x}, t) Q_{j}(\vec{y}, t)\right| P>=\psi_{i j}^{(\xi)}(\vec{x}-\vec{y} ; P) e^{-i P \cdot X_{\xi}},
$$

where $X_{\xi}(=\xi x+(1-\xi) y)$ denotes the position of the heavy-light meson and $\xi$ is a free parameter. If we set $\xi=0$ or 1 , then $X_{\xi}$ coincides with the position of the heavy quark or light quark, respectively. With the initial and final phase factors as well as a phase from the plane wave function of the decaying particle, we need to correctly formulate the Lorentz invariant decay amplitude.

As described in detail in Ref. [2], the decay amplitude, independent of $\xi$ as expected, can be given as

$$
\begin{aligned}
\mathscr{M}_{P \rightarrow P^{\prime}}= & \int d^{4} x<P^{\prime}, k\left|\mathscr{L}_{\text {int }}(x)\right| P> \\
\approx(2 \pi)^{4} & \delta^{4}\left(P-P^{\prime}-k\right) \\
& \times \gamma^{-1} \int d^{3} x \operatorname{tr}\left[\left(G^{-1}\right)^{T} \psi_{i \ell}^{\prime \dagger}\left(\vec{x} ; M^{\prime}\right) G^{-1} O_{i j} G \psi_{j \ell}(\vec{x} ; M) G^{T}\right] e^{-2 i m_{Q} V z} .
\end{aligned}
$$

Here the final expression is written in terms of the rest frame wave functions, $\psi^{(\prime)}\left(\vec{x} ; M^{(\prime)}\right), G$ is a Lorentz-boost operator, $\gamma$ is a Lorentz factor, and $V$ is a velocity of both initial and final heavylight mesons in the Breit frame. Three phases coming from initial, final and decay particles give the four-momentum conservation, $\delta^{4}\left(P-P^{\prime}-k\right)$. There remains, however, one phase which comes from the recoil effects of the initial and final parcitles, $e^{-2 i m_{Q} V z}$, whose phase is equal to $-i|\vec{k}| z$ of the plane wave in the heavy quark limit but they are different in general with finite $m_{Q}$.

We assume chiral interaction of pseudoscalar mesons $(\pi$ and $K$ ) with light quarks. In the present paper we only compute one pseudoscalar particle emission from heavy-light mesons, and then the relevant interaction Lagrangian is as follows:

$$
\mathscr{L}_{\text {int }}=\frac{g}{\sqrt{2} f_{\pi}} \bar{q}_{i} \gamma_{\mu} \gamma_{5} q_{j} \partial^{\mu} \phi_{i j}
$$

where $g$ is a dimensionless coupling constant and $f_{\pi}$ is the pion decay constant. Here the flavor $S U(3)$ symmetry is assumed and $\phi_{i j}$ 's represent the octet meson fields, that is,

$$
\left(\phi_{i j}\right)=\sqrt{2}\left(\begin{array}{ccc}
\frac{1}{\sqrt{2}} \phi_{3}+\frac{1}{\sqrt{6}} \phi_{8} & \pi^{+} & K^{+} \\
\pi^{-} & -\frac{1}{\sqrt{2}} \phi_{3}+\frac{1}{\sqrt{6}} \phi_{8} & K^{0} \\
K^{-} & \bar{K}^{0} & -\frac{2}{\sqrt{6}} \phi_{8}
\end{array}\right) .
$$


The mixing of $\pi^{0}$ and $\eta$ is taken into account with a small parameter $\varepsilon$ as follows:

$$
\left(\begin{array}{c}
\pi^{0} \\
\eta
\end{array}\right)=\frac{1}{\sqrt{1+\varepsilon^{2}}}\left(\begin{array}{cc}
1 & \varepsilon \\
-\varepsilon & 1
\end{array}\right)\left(\begin{array}{l}
\phi_{3} \\
\phi_{8}
\end{array}\right) .
$$

This is used to calculate decay widths of $D_{s 0}(2317)$ and $D_{s 1}(2460)$, which are below the $D K^{*}$ thresholds so that we need the isospin breaking term, i.e., $\pi^{0}-\eta$ mixing.

\section{Form Factors}

In general the matrix elements of the axial-vector current between the various spin states of the heavy-light mesons have the following tensor structures,

$$
\begin{aligned}
& \frac{<0^{-}\left|j_{5 \mu}\right| 1^{-}>}{\sqrt{M_{2} M_{1}}}=(1+\omega) \varepsilon_{1 \mu} \xi_{A 1}^{(k)}(\omega) \\
& +\left(\varepsilon_{1} \cdot v_{2}\right)\left\{\left(v_{1}+v_{2}\right)_{\mu} \xi_{A 2}^{(k)}(\omega)+\left(v_{1}-v_{2}\right)_{\mu} \xi_{A 3}^{(k)}(\omega)\right\} \quad(k=1,10), \\
& \frac{<0^{-}\left|j_{5 \mu}\right| 0^{+}>}{i \sqrt{M_{2} M_{1}}}=\left(v_{1}+v_{2}\right)_{\mu} \xi_{A 1}^{(2)}(\omega)+\left(v_{1}-v_{2}\right)_{\mu} \xi_{A 2}^{(2)}(\omega), \\
& \frac{<1^{-}\left|j_{5 \mu}\right| 0^{+}>}{\sqrt{M_{2} M_{1}}}=\varepsilon_{\mu v \rho \sigma} v_{1}^{v} v_{2}^{\rho} \varepsilon_{2}^{* \sigma} \xi_{A}^{(3)}(\omega) \\
& \frac{<0^{-}\left|j_{5 \mu}\right| 1^{+}>}{\sqrt{M_{2} M_{1}}}=\varepsilon_{\mu v \rho \sigma} v_{1}^{v} v_{2}^{\rho} \varepsilon_{1}^{\sigma} \xi_{A}^{(k)}(\omega) \quad(k=4,5), \\
& \frac{<1^{-}\left|j_{5 \mu}\right| 1^{+}>}{i \sqrt{M_{2} M_{1}}}=\left(\varepsilon_{2}^{*} \cdot \varepsilon_{1}\right)\left(v_{1}+v_{2}\right)_{\mu} \xi_{A 1}^{(k)}(\omega)+\left(\varepsilon_{2}^{*} \cdot \varepsilon_{1}\right)\left(v_{1}-v_{2}\right)_{\mu} \xi_{A 2}^{(k)}(\omega) \\
& +\left(\varepsilon_{2}^{*} \cdot v_{1}\right) \varepsilon_{1 \mu} \xi_{A 3}^{(k)}(\omega)+\left(\varepsilon_{1} \cdot v_{2}\right) \varepsilon_{2 \mu}^{*} \xi_{A 4}^{(k)}(\omega) \quad(k=6,7), \\
& \frac{<0^{-}\left|j_{5 \mu}\right| 2^{+}>}{i \sqrt{M_{2} M_{1}}}=\varepsilon_{1 \mu \nu} v_{2}^{v} \xi_{A 1}^{(8)}(\omega)+\varepsilon_{1 \alpha \beta} v_{2}^{\alpha} v_{2}^{\beta}\left\{v_{1 \mu} \xi_{A 2}^{(8)}(\omega)+v_{2 \mu} \xi_{A 3}^{(8)}(\omega)\right\} \text {, } \\
& \frac{<1^{-}\left|j_{5 \mu}\right| 2^{+}>}{\sqrt{M_{2} M_{1}}}=\varepsilon_{\mu v \rho \sigma}\left[\varepsilon_{1}^{v \alpha} v_{2 \alpha} \varepsilon_{2}^{* \rho}\left\{\left(v_{1}+v_{2}\right)^{\sigma} \xi_{A 1}^{(9)}(\omega)+\left(v_{1}-v_{2}\right)^{\sigma} \xi_{A 2}^{(9)}(\omega)\right\}\right. \\
& \left.+v_{1}^{\rho} v_{2}^{\sigma}\left\{\varepsilon_{1}^{v \alpha} \varepsilon_{2 \alpha}^{*} \xi_{A 3}^{(9)}(\omega)+\varepsilon_{1}^{v \alpha} v_{2 \alpha}\left(\varepsilon_{2}^{*} \cdot v_{1}\right) \xi_{A 4}^{(9)}(\omega)+\varepsilon_{1 \alpha \beta} v_{2}^{\alpha} v_{2}^{\beta} \varepsilon_{2}^{* v} \xi_{A 5}^{(9)}(\omega)\right\}\right] \\
& +\varepsilon_{\alpha \beta \gamma \delta} \varepsilon_{1}^{\alpha \alpha^{\prime}} v_{2 \alpha^{\prime}} \varepsilon_{2}^{* \beta} v_{1}^{\gamma} v_{2}^{\delta}\left\{\left(v_{1}+v_{2}\right)_{\mu} \xi_{A 6}^{(9)}(\omega)+\left(v_{1}-v_{2}\right)_{\mu} \xi_{A 7}^{(9)}(\omega)\right\}, \\
& \frac{<1^{-}\left|j_{5 \mu}\right| 1^{-}>}{i \sqrt{M_{2} M_{1}}}=\varepsilon_{\mu \nu \rho \sigma}\left[\varepsilon_{1}^{v} \varepsilon_{2}^{* \rho}\left\{\left(v_{1}+v_{2}\right)^{\sigma} \xi_{A 1}^{(11)}(\omega)+\left(v_{1}-v_{2}\right)^{\sigma} \xi_{A 2}^{(11)}(\omega)\right\}\right. \\
& \left.+v_{1}^{\rho} v_{2}^{\sigma}\left\{\varepsilon_{1}^{v}\left(\varepsilon_{2}^{*} \cdot v_{1}\right) \xi_{A 3}^{(11)}(\omega)+\varepsilon_{2}^{* v}\left(\varepsilon_{1} \cdot v_{2}\right) \xi_{A 4}^{(11)}(\omega)\right\}\right] \\
& +\varepsilon_{\alpha \beta \gamma \delta} \varepsilon_{1}^{\alpha} \varepsilon_{2}^{* \beta} v_{1}^{\gamma} v_{2}^{\delta}\left\{\left(v_{1}+v_{2}\right)_{\mu} \xi_{A 5}^{(11)}(\omega)+\left(v_{1}-v_{2}\right)_{\mu} \xi_{A 6}^{(11)}(\omega)\right\},
\end{aligned}
$$

where the initial and final quantities have subindices 1 and 2, respectively, $\varepsilon_{i \mu}$ and $\varepsilon_{i \mu \nu}$ are polarization vector and tensor, $v_{1}$ and $v_{2}$ are velocity vectors defined by $P_{1}=M_{1} v_{1}$ and $P_{2}=M_{2} v_{2}$, which in the Breit frame have components, $v_{1}^{0}=v_{2}^{0}=\gamma, \quad v_{1}^{3}=-v_{2}^{3}=\gamma V$, and $\omega=v_{1} \cdot v_{2}=P_{1} \cdot P_{2} /\left(M_{1} M_{2}\right)$, 
which in the Breit frame is related to $V$ by

$$
\omega=\gamma^{2}\left(1+V^{2}\right)=\frac{1+V^{2}}{1-V^{2}} .
$$

In our model the $1^{+}$mass-eigenstates appearing in Eqs. (2.4) and (2.5) are characterized by the quantum number of $j=L+s_{q}$, and ${ }^{3} P_{1}$ and ${ }^{1} P_{1}$ dominant states are denoted by ${ }^{3} P_{1} "$ and ${ }^{11} P_{1} "$, respectively. This is the reason why we have two kinds of form factors in these equations, i.e., $k=4,5$ and $k=6,7$. There are also two $1^{-}$states with $k=1,10$ in Eq. (2.1) corresponding to ${ }^{3} S_{1}$ and ${ }^{3} D_{1}$ states, respectively.

\section{Numerical Results}

In order to obtain explicit forms of the form factors $\xi_{A}$ 's, we need to calculate the matrix elements by inserting the explicit forms of the wave functions. According to Eq.(1.3) the matrix elements of the axial vector current are evaluated in terms of the wave functions in the rest frame of the heavy-light mesons, $\psi_{i}(\vec{r}, M)$, as follows:

$$
\begin{aligned}
\frac{<\psi_{2}\left|j_{5}{ }^{\mu}\right| \psi_{1}>}{2 \sqrt{M_{1} M_{2}}} & \approx \gamma^{-1} \int d^{3} x \frac{1}{4 \pi r^{2}} \frac{1}{2} \operatorname{tr}\left[y_{2}^{*}\left(u_{2}, i(\vec{n} \cdot \vec{\sigma}) v_{2}\right) G^{-1}\left(\rho_{1}, \sigma_{1}, \sigma_{2}, \sigma_{3}\right) G\left(\begin{array}{c}
u_{1} \\
-i(\vec{n} \cdot \vec{\sigma}) v_{1}
\end{array}\right) y_{1}\right] e^{i q z} \\
\psi_{i}(\vec{r} ; M) & =\left(\begin{array}{c}
u_{i}(r) \\
-i(\vec{n} \cdot \vec{\sigma}) v_{i}(r)
\end{array}\right) y_{i}
\end{aligned}
$$

where $q=-2 m_{Q} V, \sigma_{i} \equiv \sigma_{i} \otimes 1_{2 \times 2}$, and $\vec{n}=\vec{r} / r$. By substitution of the explicit expression for $G$, the matrix elements of each component of the axial vector current are obtained.

After expressing the form factors $\xi_{A}$ 's in terms of the radial wave functions $u$ 's and $v$ 's, the decay widths can be calculated using the following expression,

$$
\Gamma=\frac{k_{R}}{8 \pi M_{1}^{2}} \frac{1}{2 j_{1}+1} \sum_{p o l}\left|<P_{2}, k\right| \mathscr{L}_{\text {int }}(0)\left|P_{1}>\right|^{2},
$$

where $k_{R}$ is the momentum of the chiral particle in the rest frame of the parent heavy-light meson.

The input parameters are determined to reproduce the mass spectrum of the heavy-light mesons in Refs. [3]. Their values are shown in Table 1 .

Table 1: Values of the parameters in our calculation

\begin{tabular}{|c|c|c|c|c|c|c|}
\hline$\alpha_{s}$ & $\begin{array}{c}a \\
\left(\mathrm{GeV}^{-1}\right)\end{array}$ & $\begin{array}{c}b+m_{u}\left(=m_{d}\right) \\
\left(\mathrm{MeV} / c^{2}\right)\end{array}$ & $\begin{array}{c}b+m_{s} \\
\left(\mathrm{MeV} / c^{2}\right)\end{array}$ & $\begin{array}{c}m_{c} \\
\left(\mathrm{MeV} / c^{2}\right)\end{array}$ & $\begin{array}{c}m_{b} \\
\left(\mathrm{MeV} / c^{2}\right)\end{array}$ & $g^{2}$ \\
\hline $\begin{array}{c}0.259(D) \\
0.392(B)\end{array}$ & 1.937 & 86 & 168 & 1023 & 4634 & 0.608 \\
\hline
\end{tabular}

In the following, we show some of our numerical results only in the case of excited $D$ mesons. See the whole calculations for $D_{s} / B / B_{S}$ in Ref. [2]. 
Table 2: Numerical evaluation of the decay widths of excited $D$ mesons

\begin{tabular}{|c|c|c|c|c|}
\hline Initial State $\left({ }^{2 S+1} L_{J}\right)$ & Final State & $k_{R}(\mathrm{MeV} / \mathrm{c})$ & $\Gamma_{t h}(\mathrm{MeV})$ & $\Gamma_{\text {exp }}(\mathrm{MeV})$ \\
\hline $\begin{array}{c}D^{* 0} \\
\left({ }^{3} S_{1}\right)\end{array}$ & $\begin{array}{l}D^{ \pm} \pi^{\mp} \\
D^{0} \pi^{0}\end{array}$ & $\begin{array}{c}- \\
43.1\end{array}$ & $\begin{array}{c}- \\
0.042\end{array}$ & $\begin{array}{c}\text { not allowed } \\
<1.3\end{array}$ \\
\hline $\begin{array}{l}D^{* \pm} \\
\left({ }^{3} S_{1}\right)\end{array}$ & $\begin{array}{l}D^{0} \pi^{ \pm} \\
D^{ \pm} \pi^{0}\end{array}$ & $\begin{array}{l}39.5 \\
38.2\end{array}$ & $\begin{array}{l}\text { Input } \\
\text { Input }\end{array}$ & $\begin{array}{c}0.065 \pm 0.018 \\
0.029 \pm 0.08\end{array}$ \\
\hline $\begin{array}{c}D_{0}^{*}(2400)^{0} \\
\quad\left({ }^{3} P_{0}\right)\end{array}$ & $\begin{array}{c}D^{ \pm} \pi^{\mp} \\
D^{0} \pi^{0} \\
D \pi(\operatorname{sum}) \\
\quad \text { all }\end{array}$ & $\begin{array}{l}414.2 \\
419.4\end{array}$ & $\begin{array}{c}0.99 \times 10^{2} \\
0.50 \times 10^{2} \\
2.5 \times 10^{2}\end{array}$ & $261 \pm 50$ \\
\hline $\begin{array}{c}D_{0}^{*}(2400)^{ \pm} \\
\quad\left({ }^{3} P_{0}\right)\end{array}$ & $\begin{array}{c}D^{0} \pi^{ \pm} \\
D^{ \pm} \pi^{0} \\
D \pi(\text { sum }) \\
\quad \text { all }\end{array}$ & $\begin{array}{l}461.3 \\
458.5\end{array}$ & $\begin{array}{c}1.2 \times 10^{2} \\
0.61 \times 10^{2} \\
1.8 \times 10^{2}\end{array}$ & $283 \pm 24 \pm 34$ \\
\hline$D_{0}^{*}(2400)$ & $D^{*} \pi$ & & 0 & not seen \\
\hline$D_{1}(2430)$ & $D \pi$ & & 0 & \\
\hline $\begin{array}{c}D_{1}(2430)^{0} \\
\left({ }^{3} P_{1} "\right)\end{array}$ & $\begin{array}{c}D^{* \pm} \pi^{\mp} \\
D^{* 0} \pi^{0} \\
D^{*} \pi(\operatorname{sum}) \\
\text { all }\end{array}$ & $\begin{array}{l}358.7 \\
363.1\end{array}$ & $\begin{array}{c}0.76 \times 10^{2} \\
0.39 \times 10^{2} \\
1.9 \times 10^{2}\end{array}$ & $384_{-75}^{+107} \pm 74$ \\
\hline$D_{1}(2420)$ & $D \pi$ & & 0 & not seen \\
\hline $\begin{array}{c}D_{1}(2420)^{0} \\
\left("{ }^{\prime 1} P_{1} "\right)\end{array}$ & $\begin{array}{c}D^{* \pm} \pi^{\mp} \\
D^{* 0} \pi^{0} \\
D^{*} \pi(\operatorname{sum}) \\
\text { all }\end{array}$ & $\begin{array}{l}354.5 \\
358.9\end{array}$ & $\begin{array}{l}2.7 \\
1.5 \\
6.9\end{array}$ & $20.4 \pm 1.7$ \\
\hline $\begin{array}{c}D_{1}(2420)^{ \pm} \\
\quad\left("{ }^{\prime 1} P_{1} "\right)\end{array}$ & $\begin{array}{c}D^{* 0} \pi^{ \pm} \\
D^{* \pm} \pi^{0} \\
D^{*} \pi(\operatorname{sum}) \\
\text { all }\end{array}$ & $\begin{array}{l}358.4 \\
357.0\end{array}$ & $\begin{array}{l}2.9 \\
1.4 \\
4.3\end{array}$ & $25 \pm 6$ \\
\hline $\begin{array}{c}D_{2}^{*}(2460)^{0} \\
\quad\left({ }^{3} P_{2}\right)\end{array}$ & $\begin{array}{c}D^{ \pm} \pi^{\mp} \\
D^{0} \pi^{0} \\
D^{* \pm} \pi^{\mp} \\
D^{* 0} \pi^{0} \\
D \pi+D^{*} \pi(\text { sum }) \\
\text { all }\end{array}$ & $\begin{array}{l}505.5 \\
510.2 \\
389.2 \\
393.4\end{array}$ & $\begin{array}{l}6.8 \\
3.6 \\
2.6 \\
1.4 \\
24\end{array}$ & $43 \pm 4$ \\
\hline $\begin{array}{c}D_{2}^{*}(2460)^{ \pm} \\
\quad\left({ }^{3} P_{2}\right)\end{array}$ & $\begin{array}{c}D^{0} \pi^{ \pm} \\
D^{ \pm} \pi^{0} \\
D^{* 0} \pi^{ \pm} \\
D^{* \pm} \pi^{0} \\
D \pi+D^{*} \pi(\text { sum }) \\
\text { all }\end{array}$ & $\begin{array}{l}508.4 \\
505.6 \\
391.2 \\
389.7\end{array}$ & $\begin{array}{l}7.1 \\
3.4 \\
2.7 \\
1.3 \\
15\end{array}$ & $37 \pm 6$ \\
\hline
\end{tabular}




\section{Discussion}

In the limit of $m_{Q} \rightarrow \infty$, we have discovered a new type of the sum rule as follows:

$$
\begin{aligned}
& \frac{1}{2 j+1} \sum_{p(j), p^{\prime}} \Gamma\left(|P, k, p(j)\rangle \rightarrow\left|P^{\prime}, k^{\prime}, p^{\prime}\right\rangle+\pi / K\right) \\
= & \frac{1}{2(j+1)+1} \sum_{p(j+1), p^{\prime}} \Gamma\left(|P, k, p(j+1)\rangle \rightarrow\left|P^{\prime}, k^{\prime}, p^{\prime}\right\rangle+\pi / K\right),
\end{aligned}
$$

where $P$ stands for the four-mometum of the heavy-light meson, $k$ is the eigenvalue to discriminate the spin multiplet, and $p$ and $q$ label the polarization of the total spin. The sum is taken over all states belonging to the same spin multiplet.

There are numerous studies to compute the decay widths using the Schwinger-Dyson amplitudes, for example, Ref. [4] and references therein. The coupling constant $\hat{g}$ appearing in these articles and our axial-vector coupling constant $g$ appearing in Eq. (1.4) are related to each other in the case of $D^{*} \rightarrow D+\pi$ or kinematically forbidden case of $B^{*} \rightarrow B+\pi$ as follows:

$$
\begin{aligned}
k^{\mu}\left\langle 0^{-} \pi\left|j_{5 \mu}\right| 1^{-}\right\rangle & =\left\langle H \pi \mid H^{*}\right\rangle \quad \text { (in their notation), } \\
\text { left hand side } & \approx \frac{2 g \sqrt{M_{1} M_{2}}}{f_{\pi}}\left(k^{0} \eta_{A 1}^{(1)}-k^{3} \eta_{A 2}^{(1)}\right) \varepsilon_{3} \\
& =-\frac{2 g \sqrt{M_{1} M_{2}}}{f_{\pi}} k^{3} \eta_{A 2}^{(1)} \varepsilon_{3}, \quad\left(\eta_{A 1}^{(1)}=0\right), \\
\text { right hand side } & =-\frac{2 \hat{g} \sqrt{M_{1} M_{2}}}{f_{\pi}} k^{3} \varepsilon_{3}, \\
g \eta_{A 2}^{(1)} & \approx \hat{g} .
\end{aligned}
$$

$\eta^{(1)}$ 's are defined in Eqs. (C1) and (C2) in Appendix C of Ref. [2], which are the ovelapping integrals of the initial and final wave functions. Coupling constants thus determined may be used in the study of dissociation processes $\pi+J / \psi \rightarrow D+\bar{D}$ or $\pi+\Upsilon \rightarrow B+\bar{B}$ by exchanging $D^{*}$ or $B^{*}$. We have obtained $g \eta_{A 2}^{(1)}=\sqrt{0.608} \times 0.74=0.577$ while they gave the value $\hat{g}=0.53$ using the leptonic decay of the heavy-light system. [4] Agreement of these values is not surprising because the authors of Ref. [4] as well as we have fitted couplings with the experimental data.

\section{References}

[1] J. L. Goity and W. Roberts, Phys. Rev. D60, 034001 (1999); M. Di Pierro and E. Eichten, Phys. Rev. D64, 114004 (2001).

[2] T. Matsuki and K. Seo, Phys. Rev. D 85, 014036 (2012).

[3] T. Matsuki and T. Morii, Phys. Rev. D56, 5646 (1997); T. Matsuki, T. Morii, and K. Sudoh, Prog. Theor. Phys. 117, 1077 (2007); Eur. Phys. J. A31, 701 (2007).

[4] B. El-Bennich, M. A. Ivanov and C. D. Roberts, Phys. Rev. C 83, 025205 (2011) [arXiv:1012.5034 [nucl-th]]. 